\title{
VECTOR FIELDS AND GAUSS-BONNET
}

\author{
BY PAUL F. BAUM
}

ABstract. The topic is vector-fields and characteristic classes. The starting point is the classical Gauss-Bonnet theorem and the $\mathrm{H}$. Hopf index theorem. After recalling these, curvature is used to define the Chern class of a complex analytic manifold. Then a recently proved formula relating Chern classes to zeroes of meromorphic vector-fields is given.

This expository note will briefly outline some recent developments involving zeroes of vector fields and characteristic classes. The characteristic classes used will be defined.

This really begins with the classical Gauss-Bonnet theorem [17], so recall this theorem. Let $M$ be a smooth compact oriented surface (without boundary) in $R^{3} . M \subset R^{3}$. Let $\nu$ be a smooth field of unit normal vectors on $M$.

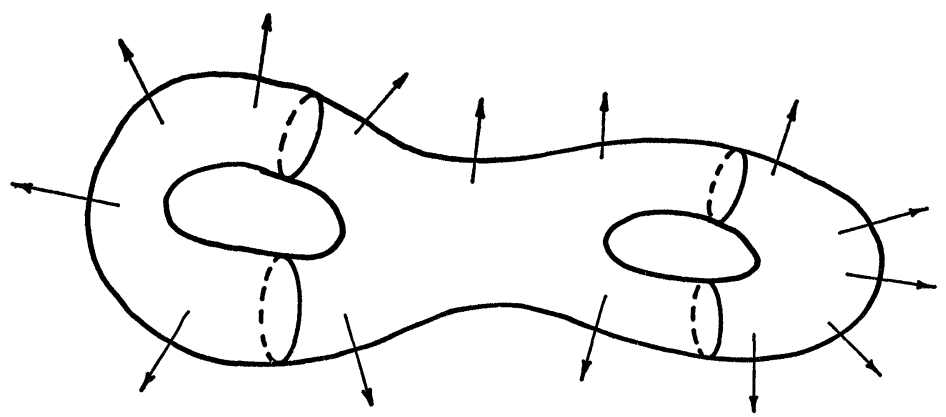

Assume that $\nu$ is compatible with the orientation of $M$ in the sense that given $p \in M$ and given a positively oriented basis $e_{1}, e_{2}$ for

$$
T_{p} M\left(T_{p} M=\text { tangent space of } M \text { at } p\right),
$$

then $\nu(p)$ is a positive multiple of $e_{1} \times e_{2}$. Let $S^{2}$ be the unit sphere of $R^{3} . S^{2}=\left\{\left(x_{1}, x_{2}, x_{3}\right) \in R^{3} \mid x_{1}^{2}+x_{2}^{2}+x_{3}^{2}=1\right\}$. Take $S^{2}$ with its standard

Based on a one-hour invited address delivered to the 74th Summer Meeting of the American Mathematical Society at Eugene, Oregon, on August 29, 1969; received by the editors June 8, 1970. Research partially supported by NSF Grants GP-6571 and GP-9580.

AMS 1970 subject classifications. Primary 57D25, 57D20, 32C10; Secondary 57D30, $53 \mathrm{C} 05$.

Key words and phrases. Gaussian curvature, Gauss-Bonnet theorem, vector-field, Hopf index theorem, complex analytic manifold, connexion, Chern class, meromorphic vector field. 
orientation. For each $p \in M$, let $g(p)$ denote the unique unit vector emanating from the origin of $R^{3}$ parallel to $\nu(p)$.

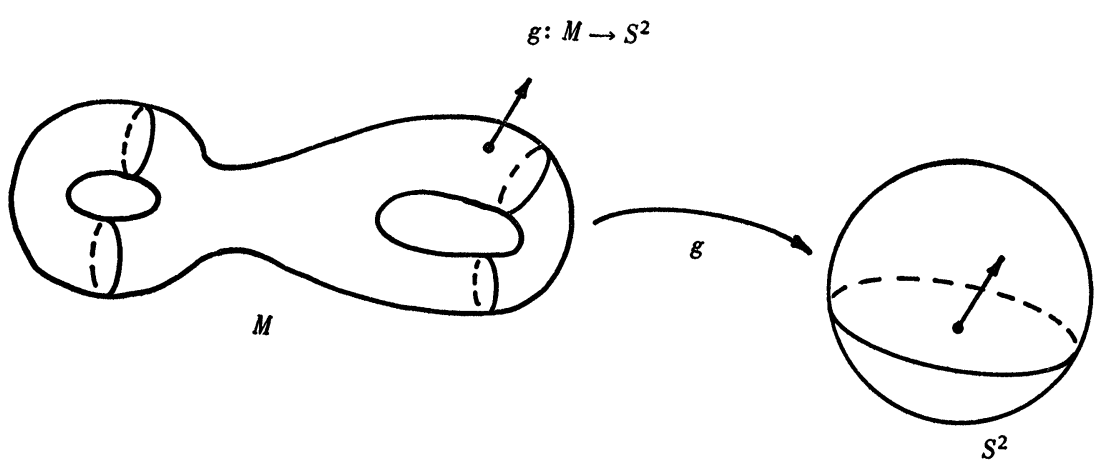

The Gaussian curvature of $M$ is defined to be the Jacobian of $g$. That is, given $p \in M$ let $A$ be a small open set of $M$ with $p \in A$. Shrink $A$ in to $p$ and define:

$$
K(p)= \pm \lim _{A \rightarrow p} \frac{\operatorname{area} g(A)}{\operatorname{area} A}
$$

where the sign is taken to be plus or minus according as to whether $g$ preserves or reverses orientation at $p$.

$K(p)$ is the Gaussian curvature of $M$ at $p . K(p)$ measures in a rather interesting fashion, the way in which $M$ is curved at $p$.

On $M$ we have the geodesics of $M$ (i.e., those curves on $M$ which locally minimize arc length). Let $\Delta$ be a triangle on $M$ whose sides are geodesics. Label the angles of $\Delta \theta_{1}, \theta_{2}, \theta_{3}$.

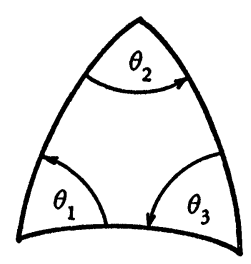

It is a theorem of Gauss that if $K$ is integrated over $\Delta$, then the number obtained is $\theta_{1}+\theta_{2}+\theta_{3}-\pi$.

$$
\int_{\Delta} K d M=\theta_{1}+\theta_{2}+\theta_{3}-\pi .
$$

(2) can be localized and globalized. 
To localize (2), let $p$ be interior to $\Delta$ and shrink $\Delta$ in to $p$, through geodesic triangles. Then it is clear from (2) that

$$
K(p)=\lim _{\Delta \rightarrow p} \frac{\theta_{1}+\theta_{2}+\theta_{3}-\pi}{\text { area } \Delta} .
$$

(3) greatly clarifies the meaning of Gaussian curvature. For one thing, (3) implies that Gaussian curvature depends only on the intrinsic differential geometry of $M$. This fact is known as the theorema egregium of Gauss. It led to the notion of curvature as defined and studied in present-day Riemannian geometry. Secondly, (3) shows that the Gaussian curvature at a point $p$ is positive or negative according as to whether small geodesic triangles about $p$ have angle sum greater than or less than $\pi$.

To globalize (2), triangulate $M$ by a triangulation in which each face is a geodesic triangle. Summing (2) over all the faces then gives that the integral of $K$ over all of $M$ is $2 \pi$ times the Euler number of $M$.

$$
\frac{1}{2 \pi} \int_{M} K d M=\chi(M)
$$

(4) is the Gauss-Bonnet theorem for $M . \chi(M)$ is by definition equal to $\beta_{0}-\beta_{1}+\beta_{2}$ where $\beta_{i}$ is the $i$ th Betti number of $M$.

(4) is really quite surprising. If $M$ is deformed a bit, then the Gaussian curvature changes radically. But despite this the integral of the Gaussian curvature over $M$ remains constant.

Very closely related to (4) is Poincaré's theorem on the zeroes of vector fields [12], [14]. Let $M$ be as above and let $V$ be a smooth tangent vector field on $M$. Assume that there are only finitely many points of $M$ where $V$ vanishes. Given $p \in M$ with $V(p)=0$ define $\omega(V, p)$ to be the winding number of $V$ about $p$. That is, introduce a local coordinate system $x_{1}, x_{2}$ about $p$ with $0=x_{1}(p)=x_{2}(p) . V$ then has the local expanison

$$
V=f_{1} \frac{\partial}{\partial x_{1}}+f_{2} \frac{\partial}{\partial x_{2}}
$$

Let $\Gamma$ be a small circle about the origin and map $\Gamma$ into $R^{2}$ by $\left(x_{1}, x_{2}\right) \rightarrow\left(f_{1}\left(x_{1}, x_{2}\right), f_{2}\left(x_{1}, x_{2}\right)\right) . \omega(V, p)$ is then the net number of times that this map winds $\Gamma$ around the origin. Poincaré's theorem asserts

$$
\sum_{p \in \operatorname{Zero}(V)} \omega(V, p)=\chi(M) \text {. }
$$

(4) and (5) imply each other.

Both the Gauss-Bonnet theorem and the Poincare theorem generalize to manifolds of higher dimension. 
If $M$ is a smooth compact oriented manifold (without boundary) and $V$ is a (tangent) vector field on $M$ with only finitely many zeroes, then according to a well-known theorem of Heinz Hopf [15], [16],

$$
\sum_{p \in \operatorname{Zero}(V)} \omega(V, p)=\chi(M) .
$$

In (6), $\omega(V, p)$ is the local degree of $V$ about $p$. This is defined by first introducing local coordinates $x_{1}, x_{2}, \cdots, x_{n}$ about $p$, with $0=x_{1}(p)=\cdots=x_{n}(p) . V$ then has the local expansion

$$
V=f_{1} \frac{\partial}{\partial x_{1}}+f_{2} \frac{\partial}{\partial x_{2}}+\cdots+f_{n} \frac{\partial}{\partial x_{n}} .
$$

Let $\Gamma$ be a small sphere of radius $r$ centered at the origin. Map $\Gamma$ to itself by

$$
x=\left(x_{1}, \cdots, x_{n}\right) \rightarrow r\left(\sum_{i=1}^{n} f_{i}(x)^{2}\right)^{-1 / 2}\left(f_{1}(x), f_{n}(x)\right) .
$$

$\omega(V, p)$ is the degree of this map in the sense of algebraic topology. More simply, $p$ is said to be a non-degenerate zero if the matrix of partial derivatives $\left\|\left(\partial f_{i} / \partial x_{j}\right)(p)\right\|$ is non-singular. In this case $\omega(V, p)$ is \pm 1 according as to whether

$$
\operatorname{det}\left\|\frac{\partial f_{i}}{\partial x_{j}}(p)\right\|
$$

is positive or negative. This is the generic case.

In (6), $\chi(M)$ is the Euler number of $M$. By definition this is $\sum_{i=0}^{n}(-1)^{i} \beta_{i}$ where $\beta_{i}$ is the $i$ th Betti number of $M$ and $n$ is the dimension of $M$.

As for Gauss-Bonnet, if $M$ is a compact oriented even-dimensional Riemann manifold, then the curvature of $M$ in the sense of Riemannian geometry determines a differential form $Q$ (called the Gauss-Bonnet form) [9] with the property:

$$
\frac{(-1)^{n / 2-1}}{2^{n} \pi^{n / 2}(n / 2 !)} \int_{M} Q=\chi(M), \quad n=\operatorname{dim} M .
$$

(6) and (7) are very closely related. When $M$ is even-dimensional, (6) and (7) imply each other. When $n=2, Q=2 K d M$.

To carry matters further, let $M$ now be a compact complex analy tic manifold with $\operatorname{dim}_{\mathrm{c}} M=n$. In the cohomology of $M$ we have the Chern classes $c_{1}, c_{2}, \cdots, c_{n}$ of $M . c_{i} \in H^{2 i}(M)$. To define these, work 
with the complex-valued smooth differential forms on $n$. So we have the de Rham complex of these forms

$$
\Lambda^{0} \stackrel{d}{\rightarrow} \Lambda^{1} \stackrel{d}{\rightarrow} \Lambda^{2} \stackrel{d}{\rightarrow} \ldots \stackrel{d}{\rightarrow} \Lambda^{2 n} .
$$

$\Lambda^{0}$ is just the collection of all smooth functions $f: M \rightarrow C$. If $z_{1}, \cdots, z_{n}$ is a local analytic coordinate system on $M$, then an $\omega \in \Lambda^{1}$ is locally of the form

$$
\sum_{i=1}^{n} f_{i} d z_{i}+\sum_{i=1}^{n} g_{i} d \bar{z}_{i}
$$

where $f_{i}$ and $g_{i}$ are smooth complex-valued functions. Similarly for $\Lambda^{2}, \Lambda^{3}, \ldots, \Lambda^{2 n}$. Note that $\Lambda^{0}$ is a ring and each $\Lambda^{i}$ is a module over $\Lambda^{0}$.

For each $p \in M$, let $T_{p} M$ be the tangent space of $M$ at $p$ viewed as a vector space over the complex numbers. $\operatorname{dim}_{\mathrm{c}} T_{p} M=n=\operatorname{dim}_{\mathrm{c}} M$. Set $T=\cup_{p \in M} T_{p} M$. $T$ has the structure of a holomorphic vector bundle over $M$ and is the holomorphic tangent bundle of $M$. Let $C^{\infty}(T)$ be the space of all smooth sections of $T$. Thus $C^{\infty}(T)$ is the space of all smooth vector fields on $M . C^{\infty}(T)$ is a module over $\Lambda^{0}$, since given $f: M \rightarrow C$ and given $V \in C^{\infty}(T)$, we can form $f V \in C^{\infty}(T)$.

A connection for $T$ is a $C$-linear map $D$

$$
D: C^{\infty}(T) \rightarrow \Lambda^{1} \otimes_{\Lambda^{0}} C^{\infty}(T)
$$

such that $D(f V)=d f \otimes V+f(D V)$.

Connections are easy to construct. First one observes that locally there is no difficulty. Then one constructs a connection for $T$ on all of $M$ by patching together locally defined connections with a smooth partition of unity.

So fix a connection $D$ for $T$.

Let $U$ be an open set of $M$ and let $V_{1}, V_{2}, \ldots, V_{n}$ be a frame of $T$ on $U$, i.e., for each $p \in U, V_{1}(p), \ldots, V_{n}(p)$ is a basis (over C) for $T_{p} M$. Then on $U$ an $n \times n$ matrix $\left(\theta_{i j}\right)$ of 1 -forms is determined by the equation:

$$
D V_{i}=\sum_{j=1}^{n} \theta_{i j} \otimes V_{j} .
$$

Set $K=d \theta-\theta \wedge \theta$, i.e., $K_{i j}=d \theta_{i j}-\sum_{\alpha=1}^{n} \theta_{i \alpha} \wedge \theta_{\alpha j}$. $K$ is an $n \times n$ matrix of 2 -forms. $K$ is called the curvature matrix of $D$ with respect to the frame $V_{1}, \ldots, V_{n}$.

Let $\tilde{V}_{1}, \ldots, \tilde{V}_{n}$ be another frame of $T$ over $U$, and let $\tilde{K}$ be the 
curvature matrix of $D$ with respect to $\tilde{V}_{1}, \cdots, \tilde{V}_{n}$. Define an $n \times n$ matrix $A$ of functions by:

$$
\tilde{V}_{i}=\sum_{j=1}^{n} A_{i j} V_{j}
$$

Then $\tilde{K}=A K A^{-1}$.

This is a very agreeable equation because if we now take $\operatorname{det} K$, trace $K$, etc., then we will obtain a well-defined differential form on all of $M$. So we set

$$
\operatorname{det}\left(I+\frac{i}{2 \pi} K\right)=1+\omega_{1}+\omega_{2}+\cdots+\omega_{n} .
$$

Here $I$ is the $n \times n$ identity matrix. Note that $\omega_{1}=(i / 2 \pi)$ Trace $K$, $\omega_{n}=(i / 2 \pi)^{n} \operatorname{det} K, \omega_{i} \in \Lambda^{2 i}$. It can now be proved that each $\omega_{i}$ is closed and that the cohomology class determined by $\omega_{i}$ is independent of the choice of connection [8]. This cohomology class is, by definition, the $i$ th Chern class of $M$.

$$
c_{i}=\operatorname{class}\left(\omega_{i}\right) \in H^{2 i}(M ; \mathbf{C}) .
$$

This is the curvature definition of the Chern class, due to S. S. Chern and A. Weil.

The Gauss-Bonnet theorem [8] in the present context becomes:

$$
\int_{M} c_{n}=\chi(M)
$$

What about a Gauss-Bonnet theorem for the other $c_{i}$ ? This can be done. First recall that on $M$ we have the Poincaré duality isomorphism between the $i$ th cohomology group of $M$ and the $(2 n-i)$ th homology group of $M$.

$$
H^{i}(M ; \mathrm{C}) \cong H_{2 n-i}(M ; C) .
$$

This is a canonical isomorphism and can be explained as follows: On $M$ we have two canonical non-singular bilinear pairings.

$$
\begin{gathered}
H^{2 n-i}(M ; \mathrm{C}) \times H^{i}(M ; \mathrm{C}) \rightarrow \mathrm{C}, \\
H^{2 n-i}(M ; \mathrm{C}) \times H_{2 n-i}(M ; \mathrm{C}) \rightarrow \mathrm{C} .
\end{gathered}
$$

The upper pairing is given by $(\omega, \eta) \rightarrow \int_{M} \omega \wedge \eta$ where $\omega \in \Lambda^{2 n-i}$ and $\eta \in \Lambda^{i}$. The lower pairing is given by $(\omega, z) \rightarrow \int_{z} \omega$ where $\omega \in \Lambda^{2 n-i}$ and $z$ is a $(2 n-i)$-cycle on $M$. Since the pairings are both non-singular and have the same left term this gives an isomorphism of the right terms. 
If $V$ is a vector field on $M$ with only finitely many zeroes, then assigning to each $p \in \operatorname{Zero}(V)$ the integer $\omega(V, p)$ gives a 0 -cycle on $M$ which shall be denoted by $Z(V)$. Then in view of the Hopf theorem, (10) can be restated as

$$
c_{n}=\text { Poincaré dual of } Z(V) .
$$

Consider now $c_{i}$. Let $V_{1}, V_{2}, \ldots, V_{n-i+1}$ be $n-i+1$ smooth vector fields on $M$ in general position (i.e., make a generic choice on $n-i+1$ smooth vector fields $M$ ). At most points of $M, V_{1}, \cdots, V_{n-i+1}$ will be linearly independent (over $C$ ). But the points of $M$ at which $V_{1}, \ldots, V_{n-i+1}$ are linearly dependent (over C) will form a cycle of dimension $2(n-i)$. Hence we have a homology class $Z\left(V_{1}, V_{2}, \ldots, V_{n-i+1}\right) \in H_{2 n-2 i}(M ; C)$. The Gauss-Bonnet formula for $c_{i}$ then becomes

$$
c_{i}=\text { Poincaré dual of } Z\left(V_{1}, \ldots, V_{n-i+1}\right), \quad i=1,2, \ldots, n .
$$

(13) is usually referred to as the equivalence of the curvature definition and the obstruction theory definition of the Chern class [10], [11], [13].

(13) strongly suggests that the Chern classes of $M$ are essentially topological invariants of $M$ and depend only very weakly on the complex analytic structure of $M$. This is, in fact, the case. For instance, if the complex analytic structure of $M$ is deformed, keeping the underlying $C^{\infty}$-structure of $M$ unchanged, then the Chern classes of $M$ remain unchanged.

So up to now the complex analy tic structure of $M$ has not been used in any really essential way. At this point Raoul Bott entered the picture and did something which really did depend on the holomophic structure of $M$. Bott asked: What happens in the Hopf theorem if $V$ is a holomorphic vector field? He then proved a very interesting refinement of the Hopf theorem.

Before stating Bott's theorem we need a remark about holomorphic vector fields. Let $V$ be a holomorphic vector field on $M$ and let $p$ be a zero of $V$. Introduce analytic coordinates $z_{1}, \ldots, z_{n}$ about $p$. Then about $p, V$ has the local expansion $V=\sum_{i=1}^{n} f_{i}\left(\partial / \partial z_{i}\right)$, where the $f_{i}$ are holomorphic functions. Form the matrix $\left\|\left(\partial f_{i} / \partial z_{j}\right)(p)\right\|$. This is an $n \times n$ matrix of complex numbers. Let $\lambda_{1}, \cdots, \lambda_{n}$ be the eigenvalues of this matrix. The remark we need is that these eigenvalues $\lambda_{1}, \ldots, \lambda_{n}$ do not depend on the choice of coordinate system about $p$. Thus these eigenvalues are well-defined invariants of the local behavior of $M$ about $p . p$ is a non-degenerate zero if all the $\lambda_{i}$ are non-zero. A non-degenerate zero must be isolated.

Bott's theorem [5], [6] is: 
TheOREM (BotT). Let $V$ be a holomorphic vector field on $M$. Assume that each zero of $V$ is non-degenerate. Let $X_{1}, \ldots, X_{n}$ be indeterminates and let $\phi\left(X_{1}, \ldots, X_{n}\right)$ be a symmetric polynomial which is homogeneous of degree n. $\phi\left(X_{1}, \ldots, X_{n}\right) \in C\left[X_{1}, \ldots, X_{n}\right]$. Set $\phi\left(X_{1}, \ldots, X_{n}\right)$ $=\tilde{\phi}\left(\sigma_{1}, \ldots, \sigma_{n}\right)$ where $\sigma_{i}$ are the elementary symmetric functions of the $X_{i}$. Then

$$
\sum_{p \in Z_{\text {ero }}(V)} \frac{\phi\left(\lambda_{1}, \lambda_{2}, \ldots, \lambda_{n}\right)}{\lambda_{1} \lambda_{2} \ldots \lambda_{n}}=\int_{M} \tilde{\phi}\left(c_{1}, c_{2}, \ldots, c_{n}\right) .
$$

If $\phi=X_{1} X_{2} \cdots X_{n}$ then (14) becomes

$$
\sum_{p \in \operatorname{Zero}(V)} 1=\int_{M} c_{n} .
$$

Since the local degree of a holomorphic vector field at a non-degenerate zero is always $+1,(15)$ follows from the Hopf theorem. But in (14), $\phi$ can be any symmetric polynomial which is homogeneous of degree $n$. Hence (14) can be viewed as a greatly strengthened Hopf theorem.

V. Guillemin first proved some special cases of (14) as a corollary of the Atiyah-Bott fixed point theorem [1], [5]. Bott then followed up Guillemin's idea and gave a simple direct proof of (14) using Stokes' theorem and the curvature definition of the Chern class. Thus Bott's proof was in the Gauss-Bonnet spirit. A quite different proof of (14) has been given by M. Atiyah and I. Singer [2].

The beauty of (14) is slightly flawed by the fact that holomorphic vector fields very rarely exist on compact complex manifolds. Most compact complex manifolds do not admit any holomorphic vector fields. Bott and I, however, found that (14) can be generalized to meromorphic vector fields. This removes the flaw because meromorphic vector fields are plentiful.

In order to generalize (14) to the case when $V$ is a meromorphic vector field on $M$, the pole of $V$ must be taken into account. The pole of $V$ (i.e. the set of points where $V$ blows up) will be a complex analytic subvariety of $M$ of complex dimension $n-1$. It has complex dimension $n-1$ because locally a meromorphic vector field has the expansion

$$
V=\frac{f_{1}}{g} \frac{\partial}{\partial z_{1}}+\frac{f_{2}}{g} \frac{\partial}{\partial z_{2}}+\cdots+\frac{f_{n}}{g} \frac{\partial}{\partial z_{n}}
$$

where $f_{i}$ and $g$ are holomorphic functions. Thus locally the pole is the set of points where $g$ vanishes, and this has complex dimension $n-1$. Hence the pole is a $2(n-1)$ cycle on $M$, and this gives a 
homology class $P \in H_{2 n-2}(M ; C)$. Denote the Poincare dual of $P$ by $P^{*} . P^{*} \in H^{2}(M ; \mathrm{C})$. Define cohomology classes $a_{i} \in H^{2 i}(M ; \mathrm{C})$ by:

$$
a_{i}=c_{i}+c_{i-1} P^{*}+c_{i-2}\left(P^{*}\right)^{2}+\cdots+c_{1}\left(P^{*}\right)^{i-1}+\left(P^{*}\right)^{i} .
$$

Assume now that $V$ is a meromorphic vector field on $M$ with only nondegenerate zeroes. Away from its pole $V$ is just a holomorphic vector field so, as above, at each zero there are eigenvalues $\lambda_{1}, \lambda_{2}, \ldots, \lambda_{n}$. As above let $\phi\left(X_{1}, \ldots, X_{n}\right)$ be a symmetric and homogeneous polynomial degree $n$, and set $\phi\left(X_{1}, \ldots, X_{n}\right)=\tilde{\phi}\left(\sigma_{1}, \ldots, \sigma_{n}\right)$. Bott and I [3] found that:

$$
\sum_{p \in \mathbb{Z} \text { ero }(V)} \frac{\phi\left(\lambda_{1}, \lambda_{2}, \ldots, \lambda_{n}\right)}{\lambda_{1} \lambda_{2} \ldots \lambda_{n}}=\int_{M} \tilde{\phi}\left(a_{1}, a_{2}, \ldots, a_{n}\right) .
$$

We have devised three different proofs of (16). The first proof, see [3], depends on a slightly mysterious curvature construction which has been called a Kunstgriff. The other two proofs are unpublished. One uses a blowing-up technique and the other uses a homotopy argument. All these proofs use curvature and as far as we know at the present moment these are the only proofs of (16) currently in existence. We do not know if (16) can be proved by applying the theory of [1] and [2].

After we had conjectured and proved (16), Bott made an ingenious observation [7]. He shifted attention from the meromorphic vector field $V$ itself to the flow lines of $V$. Although $V$ itself blows up at its pole, the flow lines can be continued across the pole in a regular fashion. So the flow lines yield a 1-dimensional holomorphic foliation with singularities. The singularities occur only at the zeroes of $V$. (16) may then be interpreted as an equation relating the local behavior of this foliation at its singularities to certain cohomology classes which arise from global consideration of the foliation. Bott went on from this to show that there should be a formula generalizing (16) to the case of foliations (with singularities) of dimension greater than one. Bott and I have arrived at a partial understanding of this question and we will report on it at a future date.

From complex manifolds one can move in two directions: to Riemannian geometry or to abstract algebraic geometry. In Riemannian geometry one works with Killing vector fields and Pontryagin classes. Contributions here have been made by Bott [5], Jeff Cheeger and myself [4], and Joel Pasternack [18]. See also [2] and an unpublished cobordism argument (using rational homology manifolds) of Bott and D. Sullivan. 
It seems quite probable that the proof of (16) can be translated from de Rham cohomology to sheaf cohomology. Some progress in this direction is indicated in [3]. Once this is done, (16) will become a theorem in abstract algebraic geometry where the ground field can be any algebraically closed field. So the spirit of Gauss-Bonnet penetrates even into the realm of abstract algebraic geometry.

\section{REFERENCES}

1. M. Atiyah and R. Bott, $A$ Lefschetz fixed point formula for elliptic complexes. I, Ann. of Math. (2) 86 (1967), 374-407, MR 35 \#3701.

2. M. Atiyah and I. Singer, The index of elliptic operators. III, Ann. of Math. (2) 87 (1968), 546-604. MR 38 \#5245.

3. P. Baum and R. Bott, On the zeroes of meromorphic vector fields, Essays in Topology and Related Topics, Mémoires dédiés à Georges de Rham, Springer-Verlag, Heidelberg, 1970, pp. 29-47.

4. P. Baum and J. Cheeger, Infinitesimal isometries and Pontryagin numbers, Topology 8 (1969), 173-193. MR 38 \#6627.

5. R. Bott, Vector fields and characteristic numbers, Michigan Math. J. 14 (1967), 231-244. MR 35 \#2297.

6. - , A residue formula for holomorphic vector fields, J. Differential Geometry 1 (1967), 311-330. MR $38 \# 730$.

7. - On a topological obstruction to integrability, Proc. Sympos. Pure Math., vol. 16, Amer. Math. Soc., Providence, R. I., 1970, pp. 127-132.

8. R. Bott and S. S. Chern, Hermitian vector bundles and the equidistribution of the zeroes of their holomorphic sections, Acta. Math. 114 (1965), 71-112. MR 32 \#3070.

9. S. S. Chern, $A$ simple intrinsic proof of the Gauss-Bonnet formula for closed Riemannian manifolds, Ann. of Math. (2) 45 (1944), 747-752. MR 6, 106.

10. - Characteristic classes of Hermitian manifolds, Ann. of Math. (2) 47 (1946), 85-121. MR 7, 470.

11. - Differential geometry of fibre bundles, Proc. Internat. Congress Math. (Cambridge, Mass., 1950) Amer. Math. Soc., Providence, R. I., 1952, pp. 397-411. MR 13, 583.

12. H. Flanders, Differential forms, Studies in Global Geometry and Analysis, Math. Assoc. Amer., distributed by Prentice-Hall, Englewood Cliffs, N. J., 1967, pp. 57-95. MR $35 \# 3583$.

13. P. A. Griffiths, On a theorem of Chern, Illinois J. Math. 6 (1962), 468-479. MR $26 \# 1905$.

14. N. Hicks, Notes on differential geometry, Math. Studies, no. 3, Van Nostrand, Princeton, N. J., 1965. MR 31 \#3936.

15. H. Hopf, Vectorfelder in n-dimensionalen Mannigfaltigkeiten, Math. Ann. 96 (1926), 225-250.

16. J. Milnor, Topology from the differentiable viewpoint, The University Press of Virginia, Charlottesville, Va., 1965. MR 37 \#2239.

17. B. O'Neill, Elementary differential geometry. Academic Press, New York, 1966. MR 34 \#3444.

18. J. Pasternack, Thesis, Princeton University, Princeton, N. J., 1970.

Brown University, Providence, Rhode Island 02912 